\title{
Autosomal dominant neovascular inflammatory vitreoretinopathy — a review
}

\author{
Małgorzata Kowalczyk, Robert Rejdak@ \\ Department of General Ophthalmology and Pediatric Ophthalmology Service, Medical University in Lublin, Lublin, Poland
}

\begin{abstract}
Autosomal dominant neovascular inflammatory vitreoretinopathy (ADNIV) is a hereditary autoimmune disorder of the eye caused by mutations in the CAPN5 gene. It is characterized by non-specific uveitis in the anterior chamber and vitreous leading to panuveitis, iris and retinal neovascularization, cystoid macular edema, abnormal retinal pigmentation, vitreous hemorrhage, intraocular fibrosis, membrane formation and tractional retinal detachment. ADNIV is a progressive disease leading to complete blindness despite of treatment. Confirmation is made by genetic analysis demonstrating mutations of the CAPN5 gene.
\end{abstract}

KEY WORDS: autosomal dominant neovascular inflammatory vitreoretinopathy; ADNIV; uveitis; neovascularization; proliferative vitreoretinopathy; cystoid macular edema; CAPN5; calpain-5

Ophthalmol J 2019; Vol. 4, 15-21

\section{INTRODUCTION}

Autosomal dominant neovascular inflammatory vitreoretinopathy (ADNIV) is a very rare, autoimmune uveitis belonging to inherited vitreoretinal dystrophies [1]. The disease is caused by the mutations in the CAPN5 gene (calpain-5) [2,3]. Genetic locus for ADNIV was mapped to chromosome $11 \mathrm{q} 13$ in 1992 by Stone et al. $[4,5]$. This gene encodes a protein, calpain-5, which is an intracellular calcium-activated cysteine protease $[2,3,6]$. CAPN5 is the first nonsyndromic gene for autoimmune uveitis and among the very few Mendelian autoimmune diseases where the gene is identified [6]. There is significant phenotypic variance in ADNIV causes by different mutations in CAPN5 gene. Recent genetic tests have discovered two new mutations which were described in literature in 2017 [7].

The first scientific work describing ADNIV was published in 1990 by Steven Bennett and co-workers. The authors presented symptoms and causes of the illness illustrated with an example of large group of affected 28 of 61 members of a six generation family [8]. The ADNIV occurs with a frequency of approximately one in 1000000 births and has been found worldwide [9]. The pathophysiology and immunopathology are not yet well known. The first symptoms can occur at all ages [9]. The disease is very progressive despite the treatment and leading to complete blindness.

Loss of the b-wave amplitude on electroretinography (ERG) is a characteristic feature of ADNIV disease [8].

The pathophysiology of the illness has not been known well; therefore, the condition is given a descriptive name autosomal dominant neovascular inflammatory vitreoretinopathy.

\section{DIAGNOSIS}

The diagnosis of the disease is difficult due to several causes. Firstly, ADNIV is classified as a rare disease; secondly, the first signs can occur at any age and cannot be detected in childhood; therefore, unfortunately, the diagnosis is delayed in most cases. The symptoms of the disorder mimic several much more common eye diseases, including 
non-specific uveitis, retinitis pigmentosa, rod-cone dystrophy, proliferative diabetic retinopathy and proliferative vitreoretinopathy [8]. Because of its similarity to these disorders, ADNIV patients are often misdiagnosed. The very characteristic sign of the ADNIV is a lack of systemic features unlike in some of the disorders mentioned above [1]. It is important sign in differential diagnosis.

The family history is very important because helps to recognize hereditary nature of the disease and suggests a genetic etiology. If the patient lacks a family history of ADNIV and presents with vitreous inflammation, a thorough evaluation for infectious and inflammatory etiologies should be performed [10].

Key findings in correct diagnosis are molecular genetic examination and ERG testing. ADNIV is inherited in an autosomal dominant manner. It means that each child has a $50 \%$ chance of inheriting the condition from an affected parent. It is present in every generation of affected family. The disease is caused by the mutations in CAPN5 gene localized on chromosome 11q13.

Best's vitelliform dystrophy, another disease inherited in autosomal dominant manner, is caused by the gene mutation mapped also to chromosome $11 \mathrm{q} 13$ [11]. We do not know yet, whether there are any other common features with ADNIV disease.

Different molecular genetic testing is necessary to detect mutations. If there is no possibility to conduct comprehensive genetic testing, the diagnosis of ADNIV unfortunately is delayed.

Retinal degeneration in ADNIV patients can be detected by examining fundus autofluorescence (FAF) and infrared reflectance (IR) of the fundus. The fundus displays enhanced FAF observed during mid-to-late stage ADNIV and is associated with photoreceptor or RPE cell dysfunction. IR imaging of the retina shows pigmentary changes indicative of reactive retinal pigment epithelium cells and photoreceptor degeneration [12].

Another important examination confirming the diagnosis of the disease is ERG testing. The abnormality is detectable with electroretinography very early in the course of the disease despite the normal appearing of the retina. ERG signaling defects precedes the signs of photoreceptors degeneration [13]. In early stages, we observe a reduced b-wave on ERG, later it is selective loss of the b-wave in the scotopic bright flash [3]. This indicates dysfunction of signal transmission from photoreceptors to membranes of the inner retina such as bipolar cells due to localization of CAPN5 to the photoreceptor synapse [10]. With progression of the disease there is also a reduction of the a-wave and in the last stage the ERG is extinguished; it shows non-recordable cone and rod responses due to severe photoreceptor dysfunction $[2,3]$.

Genetic analysis or ERG testing is performed in patients who do not demonstrate classic features of ADNIV or those who are younger than 30 years of age [10].

\section{SYMPTOMS}

There are 5 stages in the course of ADNIV, each lasting approximately ten years.

The first stage begins in the second or third decade of life and is asymptomatic for the patient. In the first stage we observe inflammatory cells in the vitreous, minimal far peripheral arteriolar closure, pigmentation and mild peripheral ischemia. In this stage, ADNIV is clinically indistinguishable from an autoimmune, non-infectious uveitis.

The electroretinogram demonstrates early retinal and photoreceptor dysfunction. The most characterized feature is reduction of b-wave.

In the second stage, patients become symptomatic, the anterior chamber shows mild inflammatory cells and there is early development of cataract. The inflammatory cells are still observed in the vitreous. The posterior segment shows pigmentary retinal degeneration and some macular and optic nerve head edema.

Stage three of the disease usually develops during the third or fourth decades, but in some cases can start earlier [14]. There are moderate inflammatory cells, progressive cataract and iris synechiae. Sometimes peripheral iris stromal atrophy and thinning occur. Development of band keratopathy is sometimes seen in the patients. The posterior segment shows cystoid macula edema (Fig. 1AB), progressive vascular closure with neovascularization of the far peripheral retina or optic disc. With the development of inflammation in the vitreous, epiretinal and subretinal membranes start to develop (Fig. 2AB). There are extensive pigmentary and atrophic changes throughout the fundus. Optical coherence tomography reveals distortion of the retinal layers. There is a decline of peripheral vision with progressively constricted visual field, which indicates reduced retinal light sensitivity. In some cases despite of the significant disease progression, the limited central vision is observed [14]. 
A

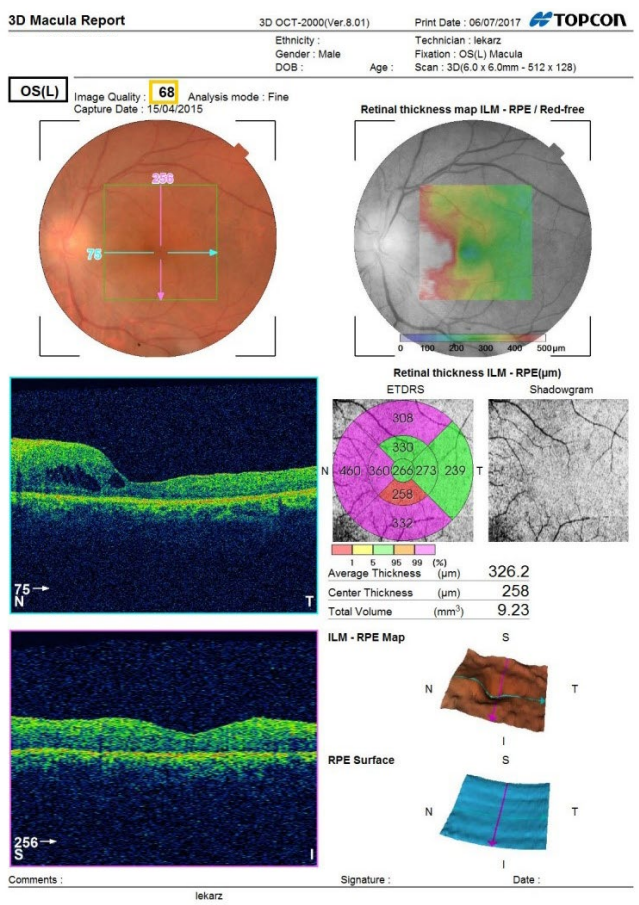

B

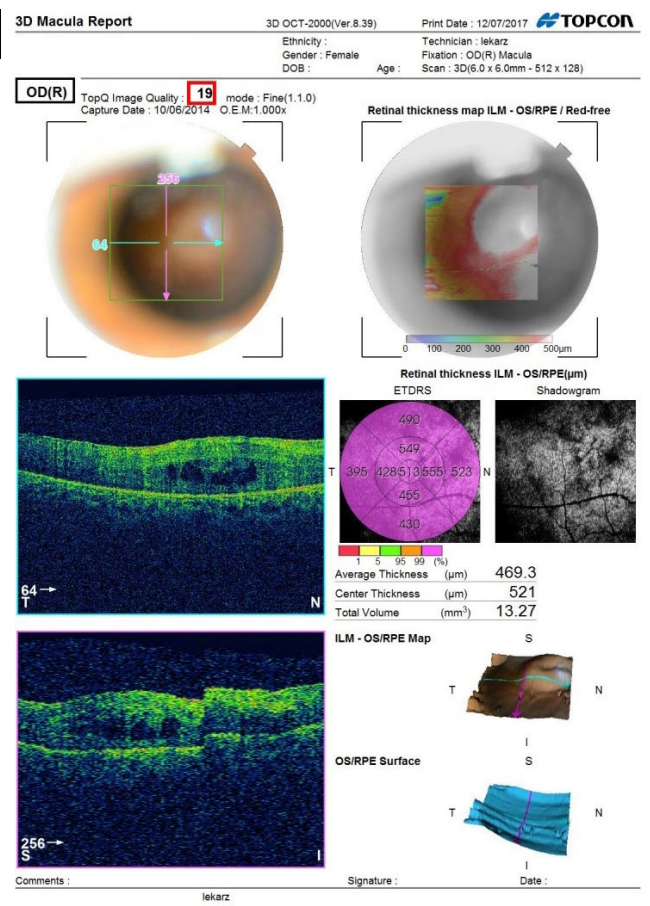

FIGURE 1AB. Optical coherence tomography (OCT) presenting cystoid macular edema (CME) in patients with autosomal dominant neovascular inflammatory vitreoretinopathy

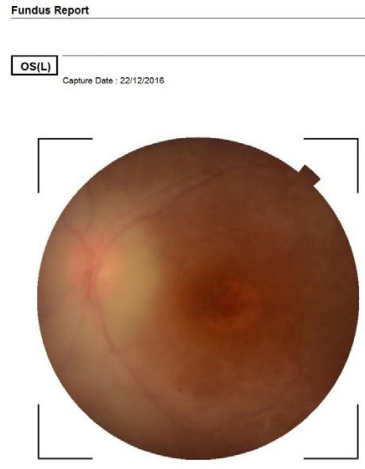

A
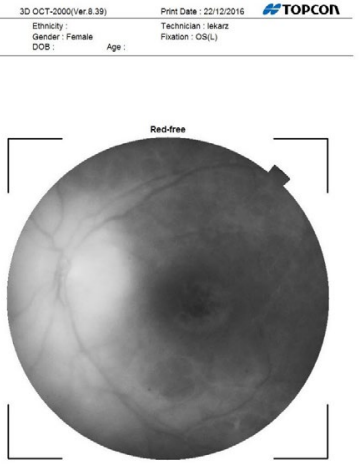

Dots:
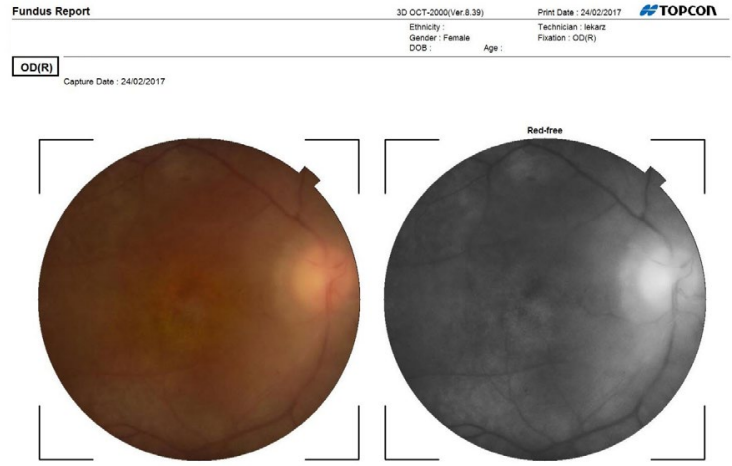

B

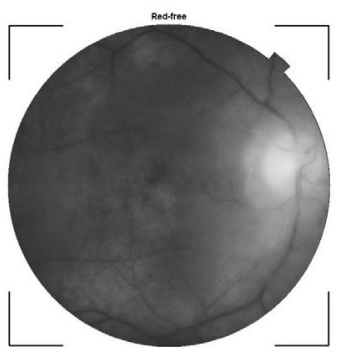

Slgneture:

FIGURE 2AB. Fundus images showing vitritis in a patient with autosomal dominant neovascular inflammatory vitreoretinopathy

In stage four neovascular and angle closure glaucoma caused by inflammation in the anterior chamber is observed; next, epiretinal membranes formation and proliferative vitreoretinopathy starts. Profound visual loss is due to ongoing neovascularization of the retina that causes vitreous hemorrhage, tractional retinal detachment and neovascular glaucoma. In the last fifth stage, the eyes become hypotonous and subsequently phthisical, leading to complete blindness.

Patients with ADNIV have night blindness only as a very late manifestation of the disease. There is no evidence for systemic autoimmune or systemic inflammatory conditions in ADNIV patients.

The development of secondary glaucoma is observed in ADNIV patients. The IOP management is connected with uveitic glaucoma, steroid-response glaucoma, angle closure glaucoma and hypotony. The chronic uveitis can cause aqueous hyposecretion, due to inflammation of the ciliary body, but this IOP-lowering effect is usually overpowered by the increased resistance to trabecular drainage from inflammatory infiltrates and endothelial dysfunction [15]. Necessary steroids therapy is the result of 
steroid-induced glaucoma. Furthermore, development of neovascularization in the anterior chamber causes angle closure glaucoma. In late stages, some ADNIV eyes become hypotonous [15]. Interestingly, some ADNIV patients do not develop elevated IOP or glaucoma despite decades of ADNIV and steroid therapy.

The rate of disease progression, specific features and vision loss vary between the affected members of a given family and is also asymmetrical between eyes. For example, some of the ADNIV patients develop blindness in one eye before the other, remaining partially sighted for years [16].

\section{PATHOPHYSIOLOGY}

Autosomal dominant neovascular inflammatory vitreoretinopathy is also an autoimmune disorder but pathophysiology and immunopathology is not well known. Immunohistological stain revealed Tcell infiltrated in ADNIV patients; hence, there is a suggestion that T-cell may play main role in immunopathology of the disease [17]. No specific antibodies were detected in ADNIV patients so far. It was noted that despite organ atrophy in the late stages of the disease, antigen reactions may still be active. The phthisis in the ADNIV eye seems to be immunologically different from that observed in eyes with phthisis from other conditions [17]. It is reported that cell-mediated rather than antibody-mediated autoimmunity may be the primary inflammatory mechanism [17]. Studies of human autopsy eyes indicated that ADNIV uveitis is primarily driven by cell-mediated immunity [12]. The vitreous biopsy revealed that interleukin-6 was elevated, suggesting a stimulated inflammatory or autoimmune response [12].

The physiologic role of calpain- 5 is not well known. Calpain- 5 was found to be located in the inner and outer segments of the photoreceptor cells and also within the outer plexiform layer [13]. The mutations alter its location inside the cells and probably its proteolytic activity or specificity. So CAPN5 could account for the various inflammatory degenerations, vascular and fibrotic phenotypes observed in ADNIV patients. Wert et al. report that the effect of the ADNIV mutation is to increase CAPN5 catalytic activity. This insight was next supported by physiologic testing of the CAPN5(R243) mutation in vivo [12]. Increased calpain activity is a feature of many eye-related pathologies including retinal degeneration, retinal hypoxia, retinitis pigmentosa, retinal detachment and glaucoma [18]. Retinal damage from these pathologies can be lessened by administering the calpain inhibitor SJA6017, but it is not known which isoform(s) exert(s) an inhibitory effect [18]. CAPN5 is widely expressed in the human body in the colon, kidney, liver, trachea, uterus, eye and brain, but the ADNIV is restricted just to the eye [13]. Retinal expression of CAPN5 may be sufficient to generate an autoimmune response. Study of CAPN5 mutations can provide insight into mechanisms of this disease and help us develop treatments for ADNIV and more common eye diseases with inflammation and neovascularization. Calpains have been implicated in the pathogenesis of different diseases including cancer, multiple sclerosis, Alzheimer's disease, cataract, diabetes and muscular dystrophy [3]. Several human neurological disorders have been associated with excess calpain activity. Wort et al. generated transgenic mice expressing human CAPN5(R243L) only in the retina and observed a clinical, histologic and molecular phenotype consistent with human ADNIV [12]. This mouse model can be used to explore protease mechanism, disease progression and possible therapies and have utility for other types of uveitis.

Uveitis in ADNIV is characterized by infiltrating $\mathrm{CD} 3+$ and CD4+ inflammatory cells in the vitreous, the uvea and the retina, suggesting a predominantly T-cell process $[13,6]$. Mutation in calpain-5 causes both early $\mathrm{CD} 3+$, T-cell migration into the retina and reduction in ERG b-wave amplitudes and later photoreceptors degeneration.

The Mahajan et al. described enucleated eye of ADNIV patient due to phthisis and pain. Examination of the enucleated eye revealed a shrunken distorted phthisical globe. The intraocular contents were grossly disorganized. A segment of optic nerve was unremarkable. The cornea was depressed, brown and opaque, anterior chamber filled with hemorrhage. The posterior segment showed a vitreous filled with a red-brown material, a detached retina and subretinal hemorrhage. The optic nerve was atrophic with replacement of axons with collagenous bands. The iridocorneal angle was occluded by fibrous membranes. The retina showed extensive degeneration and retinal pigment epithelium significant cell loss [6].

Despite organ atrophy in the late stages of disease, antigens that instigate autoimmune reactions may still be active. The ADNIV autoimmune reac- 
tion continues through end-stage disease when the eye becomes shrunken and blind [17].

The molecular mechanisms that initiate neuroinflammation are poorly understood.

Immunological mechanisms in the eye are also poorly understood.

\section{DIFFERENTIAL DIAGNOSIS}

The differential diagnosis should include autoimmune uveitis, retinitis pigmentosa, rod-cone dystrophy, proliferative vitreoretinopathy and diabetic retinopathy $[8,13,19,20]$. At different stages ADNIV mimics this diseases but most of them are characterized with systemic features otherwise then in ADNIV. Comparing neovascularization seen in proliferative diabetic retinopathy and ADNIV, significant capillary dropout or evidence of ischemia is absent in ADNIV. This suggests that a non-ischemic signaling pathway activates neovascularization. There is not a crisp demarcation line of vascular closure in the peripheral retina as can be seen in other peripheral retinal vascular diseases [8].

Retinal pigmentation in ADNIV begins in the area of vascular closure and becomes large deep round spots rather than the bone spicule pattern in the midperiphery seen in retinitis pigmentosa.

Another disease to be considered in the differential diagnosis is familial exudative vitreoretinopathy. In this condition, we observe abrupt termination of the retinal vessels at the equator. Vessels probably never develop normally. In contrast, ADNIV patients initially have normal vessels which gradually become occluded. Another difference is absence of vitreous cells and loss of the b-wave on ERG testing in patients with familial exudative vitreoretinopathy [8].

There are other conditions in which we observe a selective loss of the $b$-wave, including retinal vascular diseases such as central retinal artery or vein occlusions, quinine and methanol intoxication and siderosis. Inherited conditions in which the b-wave is affected include X-linked retinoschisis, myotonic dystrophy and forms of nonprogressive night blindness [8].

Autosomal dominant neovascular inflammatory vitreoretinopathy is a unique eye disease, because it shows pathological symptoms of several eye diseases that normally do not occur together. Recognition of familial nature of ADNIV is very helpful to diagnose properly.
The disorder is dominant, highly penetrant and displays severe and different phenotype. Phenotypic discordance may be due to epigenetic, postzygotic or environmental differences [16].

\section{TREATMENT}

Treatment of ADNIV patients is very difficult and many of them are refractory to it. One of the major reasons is a chronic, severe and non-specific autoimmune uveitis in the course of ADNIV. Another reason is iris and retina neovascularization leading to hemorrhage and fibrosis causing tractional retinal detachment and ongoing outer retinal degeneration.

Uveitis accounts for as much as $10-15 \%$ of blindness in the USA and is the fifth leading cause of vision loss in developed world. The management of uveitis is often ineffective especially in non-specific uveitis, like in ADNIV patients [14]. Immunohistopathological findings suggest that underlying ocular immune dysfunction is present. The treatment should be directed at major causes of profound vision loss such as photoreceptor degeneration, cataract, cystoid macular edema, vitreous hemorrhage, dense membrane formation leading to tractional retinal detachment, neovascular and steroid-responsive glaucoma.

Initial proceeding of ADNIV is directed to reduce active inflammation in anterior and posterior segment and prevent serious complication of ongoing inflammation and neovascularization. The therapy of ADNIV involves steroids including topical, periocular and intravitreal injections. Long term local and systemic therapies are limited because of the numerous side effects like cataracts, infections and glaucoma. Periocular and intravitreal steroids require injections at regular intervals in order to control inflammation and cystoid macula edema. Some authors report the benefit of the fluocinolone acetonide (FA) implant in the treatment of chronic uveitis in ADNIV patients [14]. It provides continuous release of intraocular corticosteroid for approximately 2.5 years. It is surgically implanted through the pars plana into the vitreous and sutured to the sclera. FA implant reduces inflammation, neovascularization and, which is important, the need for systemic or local therapy, but it does not stabilize long-term vision, retinal thickening and fibrosis [21]. The corticosteroid therapy in ADNIV is a non-specific therapy. Like some other uveitic conditions, AD- 
NIV eventually becomes resistant to conventional steroid immunosuppression.

This nonspecific therapy might be optimized by medications directed at the specific mediators of ADNIV. One study suggests that therapeutic strategies targeting $\mathrm{T}$ cells may be more effective than nonspecific therapy. For example drugs such as cyclosporin A, FK06, anti-CD3 and rapamycin that target $T$ cell activation and downstream $T$ cell pathways might have greater effect than B cell drugs such as cyclosphamide and mycophenylate mofetil [17].

Treatment of ongoing iris and retinal neovascularization apart from steroids therapy includes also anti-VEGF medication. Some authors reported applying of bevacizumab for recurrent uveitis [15]. The laser photocoagulation of the peripheral retina is only partially effective. Likewise, vitrectomy surgery is related to recurrent membranes and detachments.

ADNIV patients could be candidates for retinal gene therapy, but if disease allele expression in the eye is not sufficient, therapies directed to cells outside the eye as calpain- 5 expressing T-cells might be required [13].

There is no therapy for retinal degeneration and there are no specific therapies for calpains [13]. To develop specific treatment for ADNIV an animal model is needed [13].

The development of secondary glaucoma is associated with the need of applying antiglaucoma therapy. Management of glaucoma in ADNIV patients can be challenging. Antiglaucoma medications include topical medication, surgeries like trabeculectomy, Ahmed glaucoma valve surgery. Some authors report better results performing AGV concurrent with the FA implantation [15].

Maybe earlier therapy with T-cell immunomodulators will be more successful. Specific therapy may delay or reverse symptoms in ADNIV and also in related eye diseases.

Untreated or under-treated patients have very poor prognosis.

\section{CONCLUSION}

Autosomal dominant neovascular inflammatory vitreoretinopathy is a complex hereditary autoimmune disorder. The disease management poses several challenges like diagnosis, follow-up and in particular treatment. Recognition of the condition is important because it allows proper genetic counselling and prevents unnecessary diagnostic investi- gations. Despite the aggressive treatment the visual improvement is only transient - as the disease progresses patients progressively lose vision. ADNIV patients need careful, continuous long-term follow-up to address ongoing chronic inflammation, membrane formation, neovascularization and retinal degeneration.

To better understand ADNIV pathogenesis further investigations including immunology, physiology and genetic are necessary.

Immunohistopathological findings suggest that an underlying ocular immune dysfunction is present in ADNIV patients. The creation of a pre-clinical model of ADNIV could be used to study mechanisms of ADNIV disease progression and therapy.

Further study of mechanisms of ADNIV will provide important new insight into some of the most important causes of irreversible human blindness, such as autoimmune uveitis, retinitis pigmentosa, proliferative vitreoretinopathy and diabetic retinopathy. In-depth investigation of this disorder will hopefully lead to better understanding of its pathogenesis and ultimately result in effective treatment.

\section{REFERENCES}

1. Michaelides M, Moore AT. Vitreous. In: Hoyt C, Taylor D. ed. Pediatric Ophthalmology and Strabismus. Elsevier, London 2012: 410-411.

2. Bassuk A, Yeh S, Wu S, et al. Structural Modeling of a Novel CAPN5 Mutation that Causes Uveitis and Neovascular Retinal Detachment. PLOS ONE. 2015; 10(4): e0122352, doi: 10.1371/journal. pone.0122352, indexed in Pubmed: 25856303.

3. Mahajan V, Skeie J, Bassuk A, et al. Calpain-5 Mutations Cause Autoimmune Uveitis, Retinal Neovascularization, and Photoreceptor Degeneration. PLoS Genetics. 2012; 8(10): e1003001, doi: 10.1371/ journal.pgen.1003001, indexed in Pubmed: 23055945.

4. Stone EM, Kimura AE, Folk JC, et al. Genetic linkage of autosomal dominant neovascular inflammatory vitreoretinopathy to chromosome 11q13. Human Molecular Genetics. 1992; 1(9): 685-689, doi: 10.1093/ $\mathrm{hmg} / 1.9 .685$, indexed in Pubmed: 1284594.

5. Sheffield VC, Kimura AC, Folk JC, et al. The gene for autosomal dominant neovascular inflammatory vitreoretinopathy maps to 11q13. AM J Hum Genet. 1992; 51(suppl): A35.

6. Mahajan V, Lin J. Lymphocyte infiltration in CAPN5 autosomal dominant neovascular inflammatory vitreoretinopathy. Clinical Ophthalmology. 2013: 1339, doi: 10.2147/opth.s46450, indexed in Pubmed: 23861576.

7. Randazzo NM, Shanks ME, Clouston P, et al. Two Novel CAPN5 Variants Associated with Mild and Severe Autosomal Dominant Neovascular Inflammatory Vitreoretinopathy Phenotypes. Ocul Immunol Inflamm. 2017: 1-6, doi: 10.1080/09273948.2017.1370651, indexed in Pubmed: 29040051.

8. Benett R, Folk JC, Kimura AE, et al. Autosomal Dominant Neovascular Inflammatory Vitreoretinopathy. BGSPlumX Metrics Ophthalmology. 1990; 97(9): 1125-1136.

9. MalaCards: Human Disease Database. https://www.malacards.org/.

10. Shields RA, Hao Tang P. Autosomal dominant neovascular inflammatory vitreoretinopathy (ADNIV). https://eyewiki.aao.org/Autosomal_Dominant_Neovascular_Inflammatory_Vitreoretinopathy_(ADNIV).

11. Stone EM, Nichols $B E$, Streb LM, et al. Genetic linkage of vitelliform macular degeneration (Best's disease) to chromosome 11q13. Nat Genet. 1992; 1(4): 246-250, indexed in Pubmed: 1302019. 
12. Wert K, Bassuk A, Wu WH, et al. CAPN5mutation in hereditary uveitis: the R243L mutation increases calpain catalytic activity and triggers intraocular inflammation in a mouse model. Human Molecular Genetics. 2015; 24(16): 4584-4598, doi: 10.1093/hmg/ddv189, indexed in Pubmed: 25994508.

13. Wert KJ, Skeie JM, Bassuk AG, et al. Functional validation of a human CAPN5 exome variant by lentiviral transduction into mouse retina. Human Molecular Genetics. 2013; 23(10): 2665-2677, doi: 10.1093/ hmg/ddt661.

14. Tlucek PS, Folk JC, Orien JA, et al. Fluocinolone acetonide implant inhibits neovascularization but not fibrosis in autosomal dominant neovascular inflammatory vitreoretinopathy. Arch Ophtalmol. 2013; 130(11), doi: 10.1001/archophthalmol.2012.1971, indexed in Pubmed: 22777573.

15. Cham A, Bansal M, Banda HK HK, et al. Secondary glaucoma in CAPN5-associated neovascular inflammatory vitreoretinopathy. Clin Ophthalmol. 2016; 10: 1187-1197, doi: 10.2147/OPTH.S103324., indexed in Pubmed: 27390515.

16. Rowell HA, Bassuk AG, Mahajan VB. Monozygotic twins with CAPN5 autosomal dominant neovascular inflammatory vitreoretinopathy. Clin
Ophthalmol. 2012; 6: 2037-2044, doi: 10.2147/OPTH.S40086, indexed in Pubmed: 23271883.

17. Mahajan VB, Vallone JG, Lin JH, et al. T-cell infiltration in autosomal dominant neovascular inflammatory vitreoretinopathy. Mol Vis. 2010; 16: 1034-1040, indexed in Pubmed: 20596252.

18. Schaefer $K$, Toral $M$, Velez G, et al. Calpain-5 Expression in the Retina Localizes to Photoreceptor Synapses. Invest Opthal Vis Sci. 2016; 57(6): 2509-2521, doi: 10.1167/iovs.15-18680, indexed in Pubmed: 27152965.

19. Vitreoretinopathy, neovascular inflammatory; VRNI. https://www. omim.org/entry/193235.

20. Mahajan VN, Folk JC, Fingert JH, et al. ARVO Annual Meeting. Genetic analysis and phenotypic staging of autosomal dominant neovascular inflammatory vitreoretinopathy. Invest Ophthalmol Visual Sci. 2011; 52(62): A175.

21. Tlucek PS, Folk JC, Orien JA, et al. Inhibition of neovascularization but not fibrosis with the fluocinolone acetonide implant in autosomal dominant neovascular inflammatory vitreoretinopathy. Arch Ophthalmol. 2012; 130(11): 1395-1401, doi: 10.1001/archophthalmol.2012.1971., indexed in Pubmed: 22777573. 